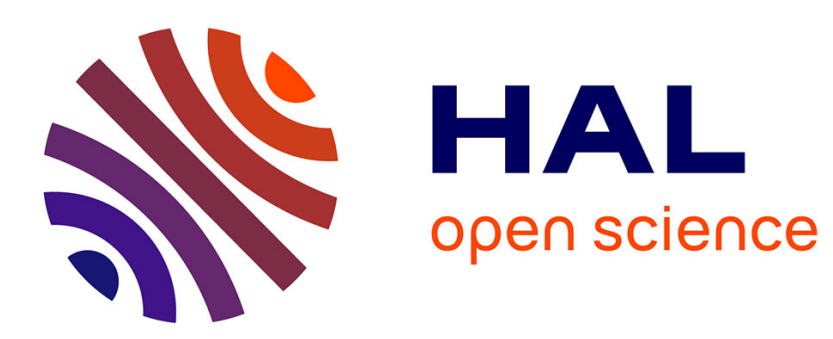

\title{
Revealing the potential of capillary electrophoresis/mass spectrometry: the tipping point
}

\author{
Rabah Gahoual, Emmanuelle Leize-Wagner, Pascal Houzé, Yannis-Nicolas \\ François
}

\section{- To cite this version:}

Rabah Gahoual, Emmanuelle Leize-Wagner, Pascal Houzé, Yannis-Nicolas François. Revealing the potential of capillary electrophoresis/mass spectrometry: the tipping point. Rapid Communications in Mass Spectrometry, 2018, 33 (S1), pp.11-19. 10.1002/rcm.8238 . hal-01981304

\section{HAL Id: hal-01981304 \\ https://hal.science/hal-01981304}

Submitted on 14 Jan 2019

HAL is a multi-disciplinary open access archive for the deposit and dissemination of scientific research documents, whether they are published or not. The documents may come from teaching and research institutions in France or abroad, or from public or private research centers.
L'archive ouverte pluridisciplinaire HAL, est destinée au dépôt et à la diffusion de documents scientifiques de niveau recherche, publiés ou non, émanant des établissements d'enseignement et de recherche français ou étrangers, des laboratoires publics ou privés. 


\section{Revealing the potential of capillary electrophoresis - mass spectrometry: the tipping point}

Rabah Gahoual ${ }^{*}$, Emmanuelle Leize-Wagner ${ }^{2}$, Pascal Houzé ${ }^{1,3}$ \& YannisNicolas François ${ }^{2}$

${ }^{1}$ Unité de Technologies Biologiques et Chimiques pour la Santé (UTCBS), Paris 5-CNRS UMR8258 Inserm U1022, Faculté de Pharmacie, Université Paris Descartes, Paris (France)

${ }^{2}$ Laboratoire de spectrométrie de masse des interactions et des systèmes (LSMIS), UDS-CNRS UMR7140, Université de Strasbourg, Strasbourg (France)

${ }^{3}$ Laboratoire de Biochimie, Hôpital Universitaire Necker-Enfants malades, Assistance Publique-Hôpitaux de Paris (AP-HP), Paris, France

Corresponding author :

Dr. Rabah Gahoual

Unité de Technologies Biologiques et Chimiques pour la Santé (UTCBS)

Faculté de Pharmacie - Université Paris Descartes

4, avenue de l'observatoire

75270 Paris Cedex 06 


\begin{abstract}
Capillary electrophoresis and mass spectrometry (CE-MS) hyphenation remains a minor technique compared to liquid chromatography - mass spectrometry (LC-MS), which represents nowadays the standard instrumentation, regardless of its introduction thirty years ago. However, from a theoretical point of view CE coupling should be quite favorable especially with electrospray ionization mass spectrometry (ESI-MS). At time, the sensitivity provided by CE-MS was often limited, due to hyphenation requirements, which at some point appeared to disqualify CE-MS from benefiting of the performance gain driving the evolution of MS instruments. However, this context has been significantly modified in a matter of a few years. The development of innovative CE-MS interfacing systems has enabled an important improvement regarding to sensitivity and reinforced robustness in order to provide an instrumentation accessible to the largest scientific community. Because of the unique selectivity delivered by the electrophoretic separation, CE-MS has revealed to be particularly relevant for the analysis of biological molecules. The conjunction of these aspects is motivating the interest of CE-MS analysis and shows that CE-MS is mature enough to enrich the toolbox of analytical techniques for the analysis of complex biological samples. Here we discuss the characteristics of the major types of high sensitivity CE-ESI-MS instrumentation and emphasize the late evolution and future positioning of CEMS analysis for the characterization of biological molecules like peptides and proteins, through some pertinent applications.
\end{abstract}




\section{Introduction}

It was consequently to the introduction of the electrospray ionization (ESI) source that the implementation of capillary electrophoresis (CE), as a separation technique prior to the mass spectrometry (MS) analysis, was investigated alongside to liquid chromatography (LC) $[1,2]$. Indeed, similarly to matrix assisted laser desorption ionization (MALDI), ESI unlocked the formation of ions from polar molecules especially biological molecules like peptides and proteins. The development of these ionization processes opened a completely unexplored field with the application of MS to the characterization of biological compounds and/or samples. As a matter of fact, we are still experiencing the outcome generated by these discoveries more than thirty years later [3]. The separation of bio(macro)molecules has been achieved for decades using electrokinetically driven separation like gel electrophoresis and CE [4]. In CE, the mobility of a compound depends on the charge in solution which is necessarily influenced by the $\mathrm{pH}$ of the background electrolyte (BGE), and the hydrodynamic radius of the compound. The difference in mobilities between the different analytes composing the sample is then leading to their separation. Because of their chemical structure, the selectivity provided by $\mathrm{CE}$ appears as particularly relevant for the separation of peptides and/or proteins which motivates its use in conjunction to MS for the analysis of biomolecules.

Since the development of the first coupling systems, CE-MS was successfully hyphenated to ESI and MALDI [1, 5]. Also it could be applied for the analysis of a large variety of biological molecules including carbohydrates, glycosylation [6], peptides [7], proteins [8]. CE has the characteristic to be a miniaturized technique. The flow of background electrolyte (BGE) represents only a few tenths $\mathrm{nL} / \mathrm{min}$ and the reduced internal diameter of the capillary - usually between 20 and $50 \mu \mathrm{m}$ - enables the formation of thin droplets at the capillary outlet. Such properties are particularly favourable to the ESI process, providing an optimal ionization efficiency and a good sensitivity [9]. However, the application of CE-MS analysis still remained quite marginal compared to the exponential development of LC-MS hyphenation which represents currently the standard instrumental approach.

In order to successfully couple CE directly to MS instrumentation, it is crucial to be able to maintain the electrical field necessary to the electrophoretic separation while the capillary outlet must be positioned 
inside the ionization source. Therefore, specific interfaces had to be designed for CE-MS systems and their implementation required some experience which greatly prevented the adoption of the technique within neophyte laboratories. In addition, the instrumental designs of early CE-MS interfaces do not necessarily provide an optimal coupling in term of ionization yields which primarily leads to a significantly limited sensitivity. Nevertheless, CE-MS hyphenation systems have recently benefited from some major instrumental improvements in order for instance to enhance the compatibility between the two techniques. This is particularly the case for CE-ESI-MS hyphenation. In the meantime, the characterization of biological molecules has demonstrated a growing interest in the analytical chemistry field which required the development of innovative methods. In this context, the characteristics of CEMS analysis in term of separation selectivity and characteristics appear particularly relevant. This trend has been emphasized by several significant publications demonstrating recently these aspects [10]. Here, we discuss the nature and impact on the performance of the latest developments regarding CEMS hyphenation. Also, we introduce several key applications over the last five years which show the outstanding capabilities of CE-MS analysis for the comprehensive analysis of bio(macro)molecules over the different structural levels in order also to understand the fields where CE-MS can make a significant contribution in the near future.

\section{Instrumental developments in CE-MS hyphenation}

Historically, a major part of CE-MS literature reports the use of sheath liquid CE-ESI-MS interfacing [1]. For this type of interface, a sheath liquid is constantly injected inside the nebulizer through a coaxial canal, external to the CE capillary (Figure 1A). The background electrolyte (BGE) and the sheath liquid are forming a junction at the extremity of the ESI nebulizer, and sprayed in a single process. The sheath liquid is connected to the $\mathrm{CE}$ outlet electrode, therefore the junction formed with the BGE enables to maintain the electric field. This type of CE-MS interface has the advantage to involve minimal specific instrumentations because the CE-MS nebulizer is designed to be positioned inside conventional ESI source. However, the ESI process needs to be performed using a grounded nebulizer to prevent any current leak between the electric field of the CE and the ESI which restricts the panel of MS instruments 
compatible with sheath-liquid CE-MS. The composition of the sheath liquid requires to be conductive in order to ensure the connectivity between CE electrodes. However it does not need to be strictly similar in composition to the BGE, which offers the possibility to use sheath liquid containing a significant proportion of organic solvent for instance to improve desolvation or a weak acid, in order to improve the protonation of the compounds even if the separation is performed using an alkaline BGE [11]. To provide the electric conductivity, the sheath liquid contains ions. Their quantity is relatively important due to a significant flow rate, that tends to limit the sensitivity due to the occurrence of ion suppression effect. These limitations can be partially tackled by careful optimization of the sheath liquid composition for each application and increasing the sample concentration [12]. However, the limitations in sensitivity systematically opposed by the sheath liquid used to prevent CE-MS from profiting to the fullest from the improvements of MS instruments regarding this particular aspect. Different instrumental alternatives were explored, in particular to alleviate the influence of the sheath liquid with the objective to provide an optimal sensitivity. Different research groups, mainly experts in separation sciences and CE, have developed their own type of CE-MS interfaces, certainly motivated by the absence of commercial alternative to sheath liquid interfaces [13]. The various CE-MS interfaces resulting from these technical improvements can be sorted in two major categories; sheath flow CE-nanoESI-MS interfaces which tend to reduce to the minimum the flow rate of sheath liquid and sheathless CE-MS interfaces that do not require a sheath liquid to enable online CE-ESI-MS hyphenation [14].

Recently, a sheath flow CE-nanoESI-MS interface was introduced by the group of Norman Dovichi. In this system, the CE capillary outlet is connected to the equivalent of a "zero dead volume" cross union. On the opposite side of the fluidic connector, a nanoESI glass emitter is positioned in order to spray directly to the MS inlet at close range. It is important to note that the CE capillary is actually hafted inside the glass emitter. The sheath liquid and the CE outlet electrode are connected to the vertical ports of the cross union (Figure 1B.). The flow rate of sheath liquid is considerably reduced, generally in the range 50-100 $\mathrm{nL} / \mathrm{min}$ range which is set in motion using the $\mathrm{CE}$ instrument through hydrodynamic or electrokinetic injection. This system allows to improve the ease of use of sheath flow CE-nanoESI-MS coupling [15]. The CE capillary and spray emitter are distinct, therefore the replacement of one or the other can be performed independently with minimal manipulations. Because the CE capillary is simply 
positioned inside the coupling system using the interface, it preserves the versatility of CE analysis like the use of coated capillaries or different internal diameters. Also this system is compatible with short length capillaries, down to $50 \mathrm{~cm}$ which in many applications is sufficient to achieve the separation due to the excellent efficiency of CE separation, and guarantee a short analysis time. With this type of interface, the glass emitter does not have to be necessarily grounded inside the source to produce a stable ESI and this characteristic has allowed to extend the range of compatible MS instruments.

The sheath flow CE-nanoESI-MS coupling could be successfully used for the analysis of complex protein digest mixtures in a shotgun proteomics strategy [16], the separation followed by top-down MS characterization of intact proteins in bacteria secretome [17] and the characterization of protein glycosylation [18]. The results described are in a general manner demonstrating an increase in MS signal intensity by 50 to 100 fold compared to conventional sheath liquid CE-MS which enabled to validate the concept of reducing the volumes of sheath liquid involved in order to enhance CE-MS sensitivity. The sheath flow CE-nanoESI-MS interface has been made commercially available recently which should allow more laboratories to be equipped and represent in the near future a formidable opportunity to push forward the analytical chemistry research implementing CE-MS analysis.

Concomitantly to the development of sheat flow CE-nanoESI-MS interfaces, different instrumental approaches were investigated to maintain the continuity of the electric field inside the CE capillary while ceasing to rely on a sheath liquid to form an electrical contact with the outlet electrode. This category is referred as sheathless CE-MS interfaces. In the case of the sheathless CE-MS interface developed by the group of Mehdi Moini, the CE capillary walls are etched using fluorhydric acid on the final 3-5 cm. This treatment creates pores in the capillary walls which allow the transfer of charges and small ions [19]. The capillary is then positioned inside a stainless steel cannula that is filed with BGE by the intermediate of a second capillary connected to the outlet electrode of the CE (Figure 1.C). Due to the porosity of the capillary walls, the electrical contact is maintained with the outlet electrode through the porous part of the capillary. The assembled cannula forms a nanoESI sprayer which can be conveniently positioned inside the source [20]. However, during sheathless CE-MS experiments only the BGE flow going out from the separation capillary is actually transferred to the MS inlet. This system allows to annihilate completely the ion suppression effect generated by the presence of a sheath liquid. As a direct 
consequence, the analysis conditions commonly used with this type of interface demonstrated a flow rate of BGE ranging from 10 to $135 \mathrm{~nL} / \mathrm{min}$, depending on the composition of the $\mathrm{BGE}$ and the eventual application of a coating [21]. In addition, the reduced internal diameter of CE capillary enables the formation of a thin electrospray composed of small droplets, similarly to nanoESI glass emitter. These two characteristics, conjugated to the absence of sheath liquid, explain the formation of a nanoESI using sheathless CE-MS interfacing which provide an optimal ionization efficiency and therefore enhances significantly CE-MS sensitivity. To accomplish this level of performance and a robustness compatible with a large number of analysis, the dimensions of the CE capillary require to be relatively standardized and the application of coatings are currently limited which reduce the versatile aspect of CE. Due to the assembling design of the cannula, the sheathless CE-MS is compatible to a large part of ESI-MS instruments.

Using this interfacing format, sheathless CE-MS could be used for instance for metabolomic analysis of urine [22], the analysis of peptide mixtures generated from protein digests in shotgun proteomic approach [23], the characterization of post-translational modifications [24], the characterization of intact proteins $[25,26]$ including top-down proteomic applications [27], and the site-specific characterization of glycosylation [28]. These results show that sheathless CE-MS offers the conservation of an optimal sensitivity between CE and MS techniques. Indeed, systematic comparison highlighted a sensitivity improvement of at least 100 fold compared to conventional sheath liquid CE-MS analysis [29, 30]. In a similar way to sheath flow CE-nanoESI-MS, sheathless CE-MS demonstrated sensitivities comparable to the current (nano)LC-MS/MS instrumentation. It has been commercially available for a few years which allowed research groups already using conventional CE-MS to have access to a highly sensitive system and explains the development of a significant number of application using this type of interface in the last few of years.

Because of the volume involved, $\mathrm{CE}$ is considered as a miniaturized technique. Thereby, recent instrumental developments report the design of CE-MS hyphenation using a microfluidic chip based system. The chip incorporates both the CE capillary and the nanoESI sprayer. Similarly to the sheath flow CE-nanoESI-MS coupling previously described, the electrical field is maintained using a low sheath flow connected to the outlet electrode which is circulating through a second channel carved into 
the chip as well. The two channels are meeting and forming a junction at the tip of the microfluidic chip close to the spraying part [31]. The sheath flow is limited to a few tenths of $\mathrm{nL} / \mathrm{min}$ which ensures an excellent sensitivity. In addition, CE separation in microfluidic channels shows an improved efficiency because of lower longitudinal diffusion attributed to the reduced internal diameter of microfluidic channels. The conventional CE instruments are not capable to operate the microfluidic chips so a dedicated instrument is necessary to use this type of CE-MS hyphenation system. However, this system has been commercially available for a few months giving access without any engineering expertise to the specific CE instrument as well as the microchip CE-MS device. This type of CE-MS interface has been recently used to develop particularly interesting applications such as the characterization of carbohydrates and glycosylations [32], the analysis of haemoglobin and the characterization of different types of intact therapeutic proteins [31, 33, 34]. This microchip CE-MS system is also commercially available as a standalone instrument including the $\mathrm{CE}$ and the interface. Indeed, because the separation capillary is directly fabricated inside the microchip, conventional CE instruments are not compatible. In a similar manner, the type of available capillaries in term of length or internal diameter for example are limited at the moment. The several types of CE-ESI-MS interfaces available are resulting from different strategies adopted to improve the sensitivity and robustness achieved in CE-MS experiments. Each type of CE-ESI-MS coupling described exhibits different interests and limitations, therefore understanding these characteristics is indubitably important in order to select the most appropriate hyphenation system for a given analysis.

The most recent instrumental developments regarding CE-MS are not only confined to interfacing efficiently both techniques. A system enabling bi-dimensional (2D) CE-ESI-MS was described in a recent work by the group of Christian Neusüß. In a fashion close to 2D liquid chromatography, this $2 \mathrm{D}$ CE-ESI-MS system uses a set of mechanical valves in order to perform two consecutives electrophoretic separations. The outlet of the second capillary was positioned inside the ESI source by the intermediate of a sheath liquid CE-MS interface. Regardless of the position of each valves, the CE capillaries extremities are always connected to the CE electrodes which prevents uncontrolled variations and/or shortage of the electrical fields or current leaks. The system could be assembled using only commercially available elements (valves, connectors) [35]. 2D CE-ESI-MS properties can be used to perform two 
consecutives electrophoretic separations in order to combine in a single analysis, two electrophoretic separation modes and improve peak capacity, likewise 2D liquid chromatography. 2D CE-ESI-MS could be used to realize in the first dimension a CIEF separation which BGE was not compatible with ESI-MS. To enable hyphenation with ESI-MS, CZE using a volatile BGE was performed in the second dimension. Because the volume of each fraction is not important, the low quantities of CIEF electrolyte were not interfering with the ESI-MS analysis [36]. In this configuration, raw separation improvements were secondary whereas the characteristic sought was the possibility to conveniently realize CIEF mobilisation followed by high resolution MS characterization. Because a large part of BGE conventionally used in CE are not volatile enough to be compatible with ESI-MS, this instrumental approach could be envisaged in the near future to use unorthodox BGE or implement other electrophoretic separation modes like micellar electrokinetic chromatography (MEKC) in conjunction with ESI-MS.

The instrumental developments concerning CE-ESI-MS hyphenation have been important over the last decade, targeting different aspects of the instrumentation. They have allowed to improve the compatibility between the two techniques in order to provide a better robustness in addition to benefit to the fullest from the outstanding sensitivity provided by late generation MS instruments. Yet one major opportunity of these technical improvements lies in the commercial availability of highly sensitive CEESI-MS hyphenation systems which gives access for an extended scientific community to this type of innovative instrumentations without requiring an important engineering background.

\section{Applications and potential of CE-MS for the analysis of biomolecules}

From a theoretical point of view, CE selectivity appears particularly relevant for the separation of biomolecules. This aspect is largely emphasized as the technical improvements described previously are in many cases applied to the analysis of biomolecules, like peptides and proteins. In parallel, the interest for the analysis of biological molecules has been constantly growing and innovative methodologies still need to be developed in order to address the challenge rising from their structural complexity. 
Peptide centric analysis using CE-ESI-MS. Recently, CE-MS(/MS) was extensively investigated for the analysis of peptide mixtures. In a bottom-up strategy, the analysis of peptide mixtures is generally used after a proteolytic digestion to identify the different proteins constituting a complex sample for example, or to perform the characterization of the primary structure of a purified protein. The last two decades have seen the advent of biopharmaceutical products, especially through the wide adoption of the monoclonal antibodies (mAbs) format. The implication of such complex glycoproteins has rapidly urged for the development of analytical methods able to give a detailed and comprehensive characterization of this type of proteins and CE-MS has been investigated in this context. Results reported have shown, using sheathless CE-MS, the possibility to achieve systematically a $100 \%$ sequence coverage in a single analysis for several therapeutic mAbs using a sample quantity corresponding to $16 \mathrm{ng}$ of digested proteins [7]. Thus, the incorporation of the electrophoretic separation demonstrated the successful migration and transfer to the MS source of a wide diversity of peptides regarding to their chemical structure in a single experiment, including hydrophilic and significantly large peptides. That characteristic participates to the improved sequence coverage and proved to be particularly interesting to obtain a consistent and highly detailed characterization of the amino acid sequence of biopharmaceutical proteins in a single experiment [37]. In particular, a similar CE-MS/MS methodology could be used to investigate the similarity between different therapeutic monoclonal antibodies (mAbs) and their respective biosimilar candidate which emphasizes the outstanding level of characterization and robustness of the data generated [38]. Also, CE-MS could also be validated through inter-laboratory studies showing the transferability and robustness of this instrumental approach [39].

High sensitivity CE-ESI-MS coupling like sheath flow CE-nanoESI-MS and sheathless CE-MS could be used for highly demanding analysis such as complex mixtures of proteins in shotgun proteomic experiments, previously restricted to (nano)LC-MS/MS, with comparable sensitivities [40]. Indeed, systematic comparisons performed in similar conditions regarding to analysis time and the quantity of sample injected show for CE-MS analysis a comparable number of identifications even in the case. In addition, the results described have highlighted a complementarity of the two instrumental approaches. For the analysis of the same peptide mixture, a significant part of the identified peptides was specific to either one of the technique which is mainly explained by their respective separation selectivity. CE-MS 
has shown an increase number of identified peptides per protein which allows to reinforce the confidence of the resulting protein identification [41]. Therefore, the conjunction of this two types of analysis could potentially give additional information resulting in a further insight regarding to the sample [42]. In complement, when the sample quantity injected was drastically reduced, below $100 \mathrm{ng}$ for complex digests, CE-MS experiments led to a higher number of identifications, peptide and protein wise [43]. This level of performance is attributed to the ionization efficiency of high sensitivity CE-ESI-MS interfaces. From an analytical standing point, this property is interesting for the analysis of precious sample available in small quantities which is also supported by the fact that CE instruments are designed to handle and inject in a robust manner a few nanoliters of sample. Thus because of these characteristics, the analysis of peptide mixtures using state-of-art CE-MS instrumentation could benefit in the near future to clinical applications like early diagnostic and the patients follow-up [44].

Post-translational modifications analysis. On another aspect, recent applications have demonstrated the relevance of CE-MS analysis for the characterization of large panel of post-translational modifications (PTMs) [45]. For instance, the results described in different research articles especially show the possibility to separate, under the influence of the CE electric field, peptides exhibiting PTMs from their intact homologous prior to their transfer to the MS. Thus, the occurrence of several types of PTMs like asparagine deamidation are modifying either the net of charge or the hydrodynamic radius of the molecule which enables their separation using the selectivity of CE, especially on the peptide level. From a MS standing point, this characteristic is also interesting because their simultaneous transfer could lead to the occurrence of competition effects during the ionization process. This effect could then result in lowered sensitivity and biased estimation of the level of modification, if not preventing completely the detection of the PTM. From a biological perspective, PTMs has been identified to be crucial due to their implication in signalling or regulation process which place CE-MS as the tool-ofchoice to study protein modifications [46].

The characterization of PTMs represents an major element in order to accurately define the primary structure of proteins. For biopharmaceutical products, that attribute is therefore considered to be crucial as some PTMs has been identified to influence significantly the pharmacological properties of the protein [47]. Simultaneously to the characterization of the amino acids sequence, CE-MS/MS could be 
used to achieve the analysis of asparagine deamidation, methionine oxidation, C-terminal glutamic acid cyclization and aspartic acid isomerization. In each case, the modified peptides could be baseline separated from the intact counterpart which allowed to detect modification levels as low as $2 \%$ [38]. Among the PTMs hotspots characterized, systematic and complete separation of homologous peptides containing different isomers of aspartic acid (Asp) which allowed using CE separation to enrich the level of characterization because the $\mathrm{m} / \mathrm{z}$ for both peptides were logically identical [24]. In addition, CEMS/MS showed the ability concomitantly as well, a highly detailed characterization of PTMs could be performed including site-specific glycosylation characterization and drug localization in the case of antibody-drug conjugates (ADCs) [37].

CE has been considered for a long time to be an excellent technique for the analysis of carbohydrates and released glycosylation [48]. Indeed, these molecules have the properties to be polar and hydrophilic, which ease their analysis in aqueous conditions, and the electrokinetic separation has demonstrated the possibility to achieve their separation with high resolution. CE-MS has been extensively used as well for the characterization of glycosylation. In the most efficient approach, glycans were released from the protein using endoglycosidase enzyme like PNGase F followed by derivatization using APTS (1aminopyrene-3,6,8-trisulfonic acid) finally completed with the CE-MS analysis [49, 50]. More recently, CE-MS/MS has been applied to the analysis of glycopeptides obtained through proteolytic digestion. Using this instrumentation setting, glycopeptides having a difference of one galactose could be partially separated while glycopeptides exhibiting a difference of one sialic acid demonstrated systematically a baseline separation [28]. That separation selectivity is definitely an excellent asset in order to improve the sensitivity and enable the detection of lowly abundant glycosylations. In addition, the MS/MS spectra allowed to identify the structure of the glycan present on the protein and localize its position on the protein by identification of the fragments corresponding to the peptide backbone (Figure 3) [51]. The recent applications suggest that CE-MS is particularly performant for the characterization of peptides. So far it has been extensively used for the characterization of therapeutic proteins however because of its properties it could be an excellent technique in discovery based experiments in order to characterize proteins undescribed purified from biological samples using a de novo sequencing approach as an example. Because of the properties of current CE-MS coupling, it is expected soon that CE-MS 
could be benefit to the study of PTMs with the opportunity to be applied in a clinical context. Indeed, it could represent a powerful tool to study variations of PTMs levels depending of the physio-pathological state. In addition, because the number of identified peptides per protein is improved, the implementation of quantitative analysis by CE-MS based on the parallel consideration of several peptides could be exploited and potentially improve the accuracy of the quantification.

Intact proteins analysis using CE-MS. Concerning therapeutic proteins, the characterization of intact proteins is particularly valuable. It allows the analysis to be conducted with minimal sample treatment, preventing the introduction of biases, in a state as close to the actual product as possible. Chromatographic separation of intact proteins, especially above $50 \mathrm{kDa}$, are limited currently to size exclusion chromatography and the charge/hydrodynamic radius separation provided by $\mathrm{CE}$ is particularly relevant from this perspective. As a consequence, different CE-MS methods demonstrating the separation and characterization using MS data directly on the intact protein were reported for various types of biopharmaceutical products [26, 52]. In a similar strategy, CE-MS was recently implemented for the characterization of mAbs and ADCs showing in particular the separation of intact charge variants. Indeed, the electrophoretic separation showed on the intact protein level, the possibility to separate isoforms exhibiting PTMs or also the separation of glycoforms [34, 53].

The conjunction of CE and MS techniques could also be successfully used for the study of protein conformational changes used as therapeutic products. These applications are particularly interesting because $\mathrm{CE}$ allowed the separation of the exact same protein adopting different conformations which is attributed to a change in hydrodynamic radius only, demonstrating the exceptional separation efficiency provided by CE even for highly complex macromolecules like proteins. In the case of Aclotine, the MS data between the separated conformers showed dissimilarities concerning the relative abundance of each detected charge state, suggesting that some protonation sites were accessible to the solvent depending of the conformation of the protein (Figure 4). That original work is particularly interesting because it illustrates a powerful analytical methodologies achievable at the moment solely using CE-MS [54, 55]. Indubitably, it is for the analysis of intact proteins that CE-MS can make the most significant contribution and the scientific community is pushing in this direction. In theory, CE separation could be even achieved in native conditions which would open the way to non-covalent complexes 
separation/study. The analysis would then profit from the excellent ionization efficiency of recent CEMS coupling. Note the recent development of affinity CE-MS abounds in this sense. Because of the potential of this instrumental approach, further developments are expected for the characterization of 2D CE-ESI-MS. On this aspect, the analytical method developments are surely profiting from the experience acquired from 2D LC-ESI-MS promising rapid improvements which should enable to cumulate the selectivity of two totally different electrophoretic separation modes followed by high resolution MS characterization.

\section{Conclusion}

CE-ESI-MS is experiencing a remarkable interest in favour of various instrumental developments which have enabled the introduction of highly sensitive CE-MS instrumentation in particular for the characterization of biological molecules like peptides and proteins. The introduction of innovative CEESI-MS have allowed to improve the compatibility between both techniques regarding to sensitivity and robustness. The abolition of sensitivity limitations has revealed the true potential of implementing an electrophoretic separation prior to MS analysis, especially for the characterization of PTMs and intact proteins. As such CE-ESI-MS is already differentiating from LC-ESI-MS methods and has the potential to enrich the panel of separation selectivity hyphenated to MS. High-end CE-ESI-MS instrumentations are now widely accessible which represents also a huge asset for a larger adoption of this technique in particular in the mass spectrometry community. The conjunction of these factors is clearly promising for the further development of CE-MS in the near future for a wide range of applications like intact proteins or glycosylation analysis.

\section{References}

1. Olivares, J.A., et al., On-line mass spectrometric detection for capillary zone electrophoresis. Analytical Chemistry, 1987. 59(8): p. 1230-1232. 
2. Lovins, R.E., et al., LIQUID CHROMATOGRAPHY - MASS-SPECTROMETRY - COUPLING OF A LIQUID CHROMATOGRAPH TO A MASS-SPECTROMETER. Analytical Chemistry, 1973. 45(8): p. 1553-1556.

3. Yamashita, M. and J.B. Fenn, Electrospray ion source. Another variation on the free-jet theme. The Journal of Physical Chemistry, 1984. 88(20): p. 4451-4459.

4. Jorgenson, J. and K. Lukacs, Capillary zone electrophoresis. Science, 1983. 222(4621): p. 266272.

5. Biacchi, M., et al., Analysis of monoclonal antibody by a novel CE-UV/MALDI-MS interface. ELECTROPHORESIS, 2014. 35(20): p. 2986-2995.

6. Balaguer, E. and C. Neusüss, Glycoprotein Characterization Combining Intact Protein and Glycan Analysis by Capillary Electrophoresis-Electrospray Ionization-Mass Spectrometry. Analytical Chemistry, 2006. 78(15): p. 5384-5393.

7. Gahoual, R., et al., Full Antibody Primary Structure and Microvariant Characterization in a Single Injection Using Transient Isotachophoresis and Sheathless Capillary ElectrophoresisTandem Mass Spectrometry. Analytical Chemistry, 2014. 86(18): p. 9074-9081.

8. Staub, A., et al., Intact protein analysis in the biopharmaceutical field. Journal of Pharmaceutical and Biomedical Analysis, 2011. 55(4): p. 810-822.

9. Wilm, M.S. and M. Mann, Electrospray and Taylor-Cone theory, Dole's beam of macromolecules at last? International Journal of Mass Spectrometry and Ion Processes, 1994. 136(2): p. 167-180.

10. Gahoual, R., et al., Cutting-edge capillary electrophoresis characterization of monoclonal antibodies and related products. Journal of Chromatography B, 2016. 1032: p. 61-78.

11. Haselberg, R., G.J. de Jong, and G.W. Somsen, Capillary electrophoresis-mass spectrometry of intact basic proteins using Polybrene-dextran sulfate-Polybrene-coated capillaries: System optimization and performance. Analytica Chimica Acta, 2010. 678(1): p. 128-134.

12. Vuorensola, K., et al., Optimization of capillary electrophoretic- electrospray ionization-mass spectrometric analysis of catecholamines. ELECTROPHORESIS, 2001. 22(20): p. 4347-4354.

13. Maxwell, E.J. and D.D.Y. Chen, Twenty years of interface development for capillary electrophoresis-electrospray ionization-mass spectrometry. Analytica Chimica Acta, 2008. 627(1): p. 25-33.

14. Yin, Y., et al., Sheathless interface to match flow rate of capillary electrophoresis with electrospray mass spectrometry using regular-sized capillary. Rapid Communications in Mass Spectrometry, 2016. 30(S1): p. 68-72.

15. Liangliang, S., et al., Over 10000 Peptide Identifications from the HeLa Proteome by Using Single-Shot Capillary Zone Electrophoresis Combined with Tandem Mass Spectrometry. Angewandte Chemie International Edition, 2014. 53(50): p. 13931-13933.

16. Zhang, Z. and N.J. Dovichi, Optimization of mass spectrometric parameters improve the identification performance of capillary zone electrophoresis for single-shot bottom-up proteomics analysis. Analytica Chimica Acta, 2018. 1001: p. 93-99.

17. Zhao, Y., et al., Capillary Zone Electrophoresis-Electrospray Ionization-Tandem Mass Spectrometry for Top-Down Characterization of the Mycobacterium marinum Secretome. Analytical Chemistry, 2014. 86(10): p. 4873-4878.

18. Qu, Y., et al., Sensitive and fast characterization of site-specific protein glycosylation with capillary electrophoresis coupled to mass spectrometry. Talanta, 2018. 179: p. 22-27.

19. Tie, C., et al., Study of the electrical connection mechanism of sheathless interface for capillary electrophoresis-electrospray ionization-mass spectrometry. Journal of Mass Spectrometry, 2012. 47(11): p. 1429-1434. 
20. Cao, P. and M. Moini, A novel sheathless interface for capillary electrophoresis/electrospray ionization mass spectrometry using an in-capillary electrode. Journal of the American Society for Mass Spectrometry, 1997. 8(5): p. 561-564.

21. Faserl, K., et al., Optimization and Evaluation of a Sheathless Capillary ElectrophoresisElectrospray Ionization Mass Spectrometry Platform for Peptide Analysis: Comparison to Liquid Chromatography-Electrospray Ionization Mass Spectrometry. Analytical Chemistry, 2011. 83(19): p. 7297-7305.

22. Zhang, W., T. Hankemeier, and R. Ramautar, Next-generation capillary electrophoresis-mass spectrometry approaches in metabolomics. Current Opinion in Biotechnology, 2017. 43: p. 17.

23. Faserl, K., et al., Enhancing Proteomic Throughput in Capillary Electrophoresis-Mass Spectrometry by Sequential Sample Injection. PROTEOMICS, 2017. 17(22): p. 1700310.

24. Gahoual, R., et al., Independent highly sensitive characterization of asparagine deamidation and aspartic acid isomerization by sheathless CZE-ESI-MS/MS. Journal of Mass Spectrometry, 2016. 51(2): p. 150-158.

25. Haselberg, R., G.J. de Jong, and G.W. Somsen, Low-Flow Sheathless Capillary Electrophoresis-Mass Spectrometry for Sensitive Glycoform Profiling of Intact Pharmaceutical Proteins. Analytical Chemistry, 2013. 85(4): p. 2289-2296.

26. Bush, D.R., et al., High Resolution CZE-MS Quantitative Characterization of Intact Biopharmaceutical Proteins: Proteoforms of Interferon- $\beta 1$. Analytical Chemistry, 2016. 88(2): p. 1138-1146.

27. Han, X., et al., Sheathless Capillary Electrophoresis-Tandem Mass Spectrometry for Top-Down Characterization of Pyrococcus furiosus Proteins on a Proteome Scale. Analytical Chemistry, 2014. 86(22): p. 11006-11012.

28. Kammeijer, G.S.M., et al., Sialic acid linkage differentiation of glycopeptides using capillary electrophoresis - electrospray ionization - mass spectrometry. Scientific Reports, 2017. 7(1): p. 3733.

29. Bonvin, G., et al., Evaluation of a sheathless nanospray interface based on a porous tip sprayer for CE-ESI-MS coupling. ELECTROPHORESIS, 2012. 33(4): p. 552-562.

30. Höcker, O., C. Montealegre, and C. Neusüß, Characterization of a nanoflow sheath liquid interface and comparison to a sheath liquid and a sheathless porous-tip interface for CE-ESIMS in positive and negative ionization. Analytical and Bioanalytical Chemistry, 2018.

31. Redman, E.A., et al., Analysis of Hemoglobin Glycation Using Microfluidic CE-MS: A Rapid, Mass Spectrometry Compatible Method for Assessing Diabetes Management. Analytical Chemistry, 2016. 88(10): p. 5324-5330.

32. Khatri, K., et al., Microfluidic Capillary Electrophoresis-Mass Spectrometry for Analysis of Monosaccharides, Oligosaccharides, and Glycopeptides. Analytical Chemistry, 2017. 89(12): p. 6645-6655.

33. Chen, C.-H., et al., Intact NIST monoclonal antibody characterization-Proteoforms, glycoforms-Using CE-MS and CE-LIF. Cogent Chemistry, 2018. 4(1): p. 1-13.

34. Redman, E.A., et al., Characterization of Intact Antibody Drug Conjugate Variants Using Microfluidic Capillary Electrophoresis-Mass Spectrometry. Analytical Chemistry, 2016. 88(4): p. 2220-2226.

35. Hühner, J. and C. Neusüß, CIEF-CZE-MS applying a mechanical valve. Analytical and Bioanalytical Chemistry, 2016. 408(15): p. 4055-4061.

36. Montealegre, C. and C. Neusüß, Coupling imaged capillary isoelectric focusing with mass spectrometry using a nanoliter valve. ELECTROPHORESIS, 2018. 39(9-10): p. 1151-1154. 
37. Said, N., et al., Structural characterization of antibody drug conjugate by a combination of intact, middle-up and bottom-up techniques using sheathless capillary electrophoresis Tandem mass spectrometry as nanoESI infusion platform and separation method. Analytica Chimica Acta, 2016. 918: p. 50-59.

38. Gahoual, R., et al., Monoclonal antibodies biosimilarity assessment using transient isotachophoresis capillary zone electrophoresis-tandem mass spectrometry. mAbs, 2014. 6(6): p. 1464-1473.

39. Wenz, C., et al., Interlaboratory study to evaluate the robustness of capillary electrophoresismass spectrometry for peptide mapping. Journal of Separation Science, 2015. 38(18): p. 32623270.

40. Sun, L., et al., Third-Generation Electrokinetically Pumped Sheath-Flow Nanospray Interface with Improved Stability and Sensitivity for Automated Capillary Zone Electrophoresis-Mass Spectrometry Analysis of Complex Proteome Digests. Journal of Proteome Research, 2015. 14(5): p. 2312-2321.

41. Ibrahim, M., et al., Improvement of Mitochondria Extract from Saccharomyces cerevisiae Characterization in Shotgun Proteomics Using Sheathless Capillary Electrophoresis Coupled to Tandem Mass Spectrometry. Journal of Chromatographic Science, 2016. 54(4): p. 653-663.

42. Wang, Y., et al., Improving the Comprehensiveness and Sensitivity of Sheathless Capillary Electrophoresis-Tandem Mass Spectrometry for Proteomic Analysis. Analytical Chemistry, 2012. 84(20): p. 8505-8513.

43. Zhu, G., et al., Single-Shot Proteomics Using Capillary Zone Electrophoresis-Electrospray Ionization-Tandem Mass Spectrometry with Production of More than 1250 Escherichia coli Peptide Identifications in a 50 min Separation. Analytical Chemistry, 2013. 85(5): p. 25692573.

44. Belczacka, I., et al., Urinary CE-MS peptide marker pattern for detection of solid tumors. Scientific Reports, 2018. 8(1): p. 5227.

45. Faserl, K., et al., Investigating capillary electrophoresis-mass spectrometry for the analysis of common post-translational modifications. ELECTROPHORESIS, 2018. 39(9-10): p. 12081215.

46. Sarg, B., et al., Comparing and Combining Capillary Electrophoresis Electrospray Ionization Mass Spectrometry and Nano-Liquid Chromatography Electrospray Ionization Mass Spectrometry for the Characterization of Post-translationally Modified Histones. Molecular \& Cellular Proteomics, 2013. 12(9): p. 2640-2656.

47. Gahoual, R., et al., Detailed Characterization of Monoclonal Antibody Receptor Interaction Using Affinity Liquid Chromatography Hyphenated to Native Mass Spectrometry. Analytical Chemistry, 2017. 89(10): p. 5404-5412.

48. Guttman, A., High-resolution carbohydrate profiling by capillary gel electrophoresis. Nature, 1996. 380: p. 461.

49. Bunz, S.-C., E. Rapp, and C. Neusüss, Capillary Electrophoresis/Mass Spectrometry of APTSLabeled Glycans for the Identification of Unknown Glycan Species in Capillary Electrophoresis/Laser-Induced Fluorescence Systems. Analytical Chemistry, 2013. 85(21): p. 10218-10224.

50. Shaheer, K., et al., On-line capillary electrophoresis/laser-induced fluorescence/mass spectrometry analysis of glycans labeled with Teal ${ }^{\mathrm{TM}}$ fluorescent dye using an electrokinetic sheath liquid pump-based nanospray ion source. Rapid Communications in Mass Spectrometry, 2018. 32(11): p. 882-888. 
51. Giorgetti, J., et al., Monoclonal antibody $N$-glycosylation profiling using capillary electrophoresis - Mass spectrometry: Assessment and method validation. Talanta, 2018. 178: p. 530-537.

52. Marie, A.-L., et al., Capillary zone electrophoresis and capillary electrophoresis-mass spectrometry for analyzing qualitative and quantitative variations in therapeutic albumin. Analytica Chimica Acta, 2013. 800: p. 103-110.

53. Belov, A.M., et al., Complementary middle-down and intact monoclonal antibody proteoform characterization by capillary zone electrophoresis - Mass spectrometry. ELECTROPHORESIS, 2018. 0(0): p. in press.

54. Marie, A.-L., et al., Characterization of conformers and dimers of antithrombin by capillary electrophoresis-quadrupole-time-of-flight mass spectrometry. Analytica Chimica Acta, 2016. 947: p. 58-65.

55. Bertoletti, L., et al., Evaluation of capillary electrophoresis-mass spectrometry for the analysis of the conformational heterogeneity of intact proteins using beta2-microglobulin as model compound. Analytica Chimica Acta, 2016. 945: p. 102-109.

56. Wojcik, R., et al., Simplified capillary electrophoresis nanospray sheath-flow interface for high efficiency and sensitive peptide analysis. Rapid Communications in Mass Spectrometry, 2010. 24(17): p. 2554-2560.

57. Schlecht, J., K. Jooß, and C. Neusüß, Two-dimensional capillary electrophoresis-mass spectrometry (CE-CE-MS): coupling $M S$-interfering capillary electromigration methods with mass spectrometry. Analytical and Bioanalytical Chemistry, 2018: p. in press. 


\section{Figures}

Figure 1. Schematic representations of the principal design of CE-ESI-MS hyphenation systems. Adapted from [37, 56]. Copyright (2016) Elsevier and copyright (2010) John Wiley and Sons, Ltd.
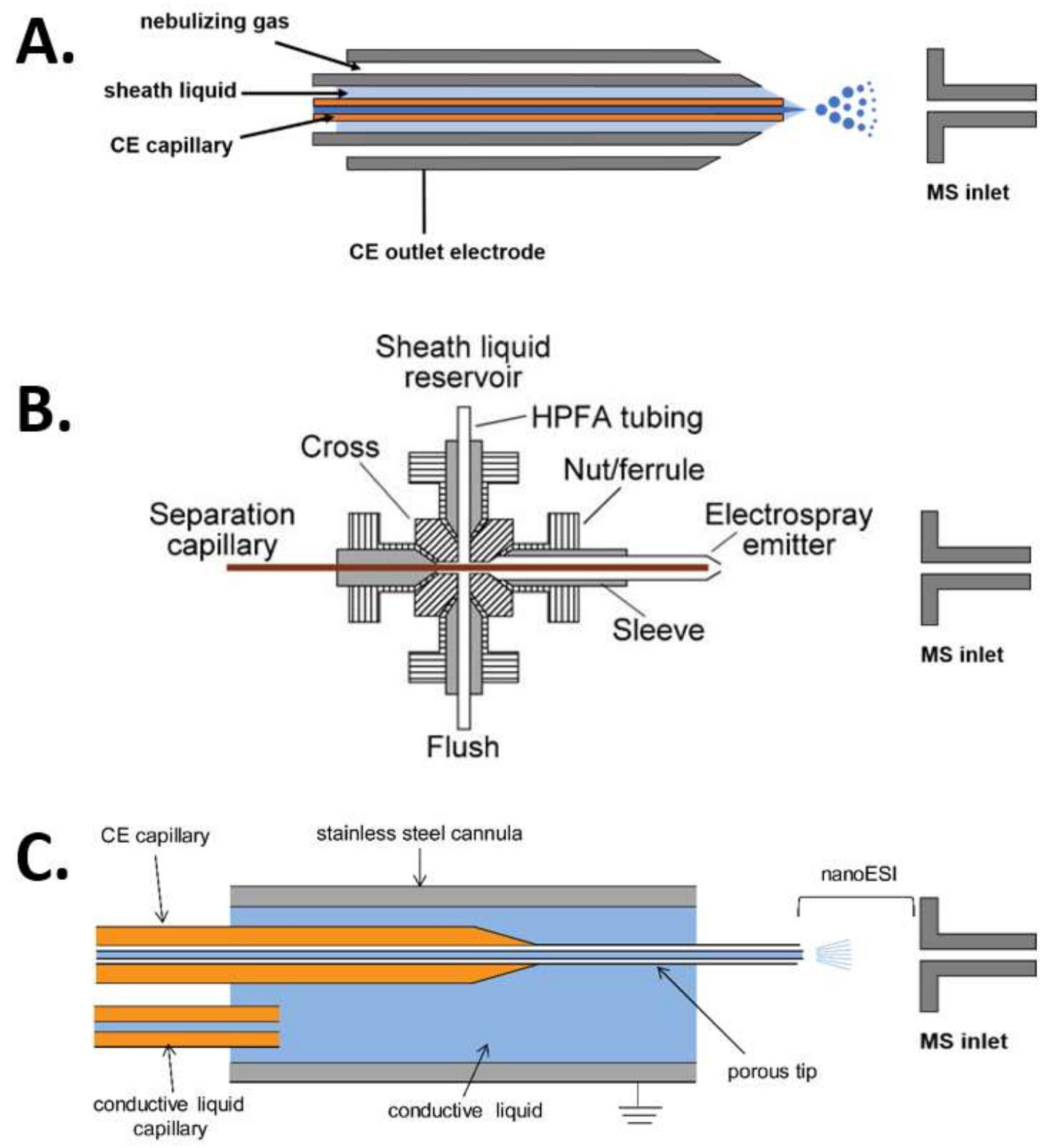
Figure 2. Schematic representations of the instrumental settings implemented to perform 2D CE-ESIMS experiments [57]. Copyright (2018) John Wiley and Sons, Ltd.

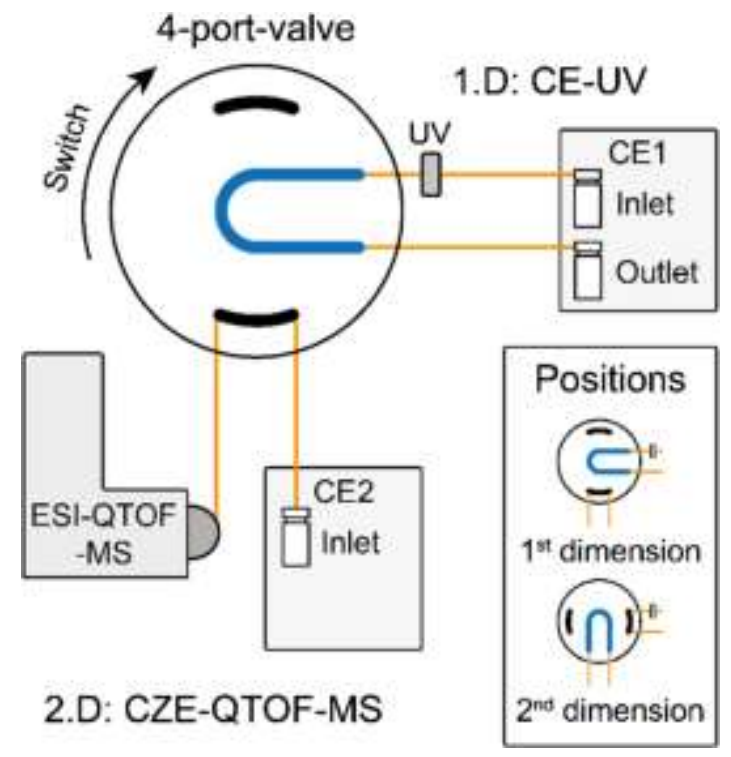

Figure 3. (a) Base Peak Electropherogram corresponding to the analysis by CE-ESI-MS/MS of natalizumab tryptic digest. (b) MS spectrum of 30.5-31.9 min and 34.9-36.5. (c) MS/MS fragmentation spectra of [EEQFNSTYR] + G0F. Adapted from [51] Copyright (2017) Elsevier B.V.
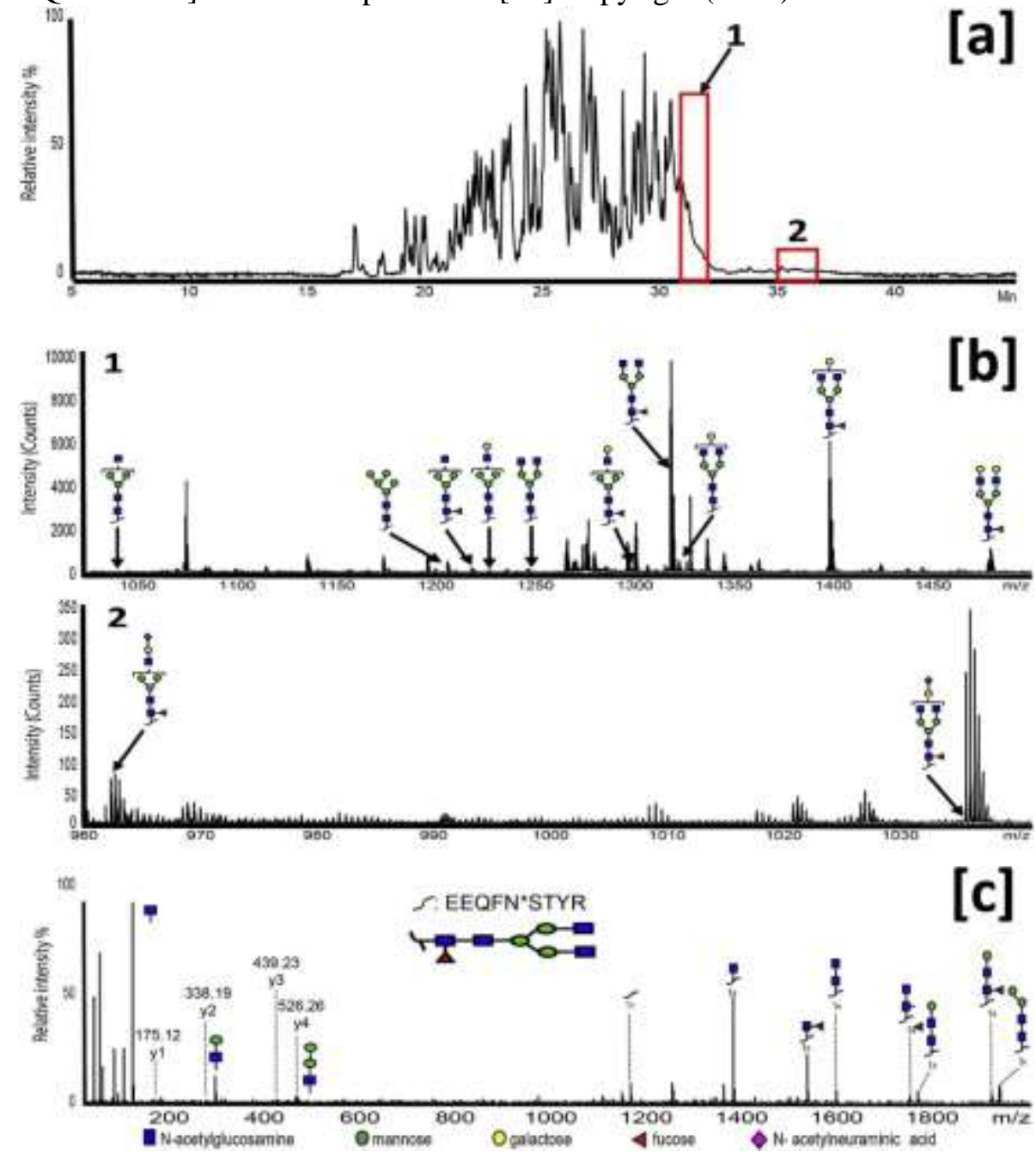
Figure 4. CE-MS of Aclotine ${ }^{\circledR}(15 \mathrm{~g} / \mathrm{L})$ using a sheath liquid of isopropanol-water 50:50 (v/v) containing $14 \mathrm{mM}$ ammonium acetate stored at room temperature for three weeks A) BPE (1500-5000 $\mathrm{m} / \mathrm{z}$ ); B) and C) mass spectra of peaks 1 and 2, respectively. Adapted from [54] Copyright (2016) Elsevier B.V.
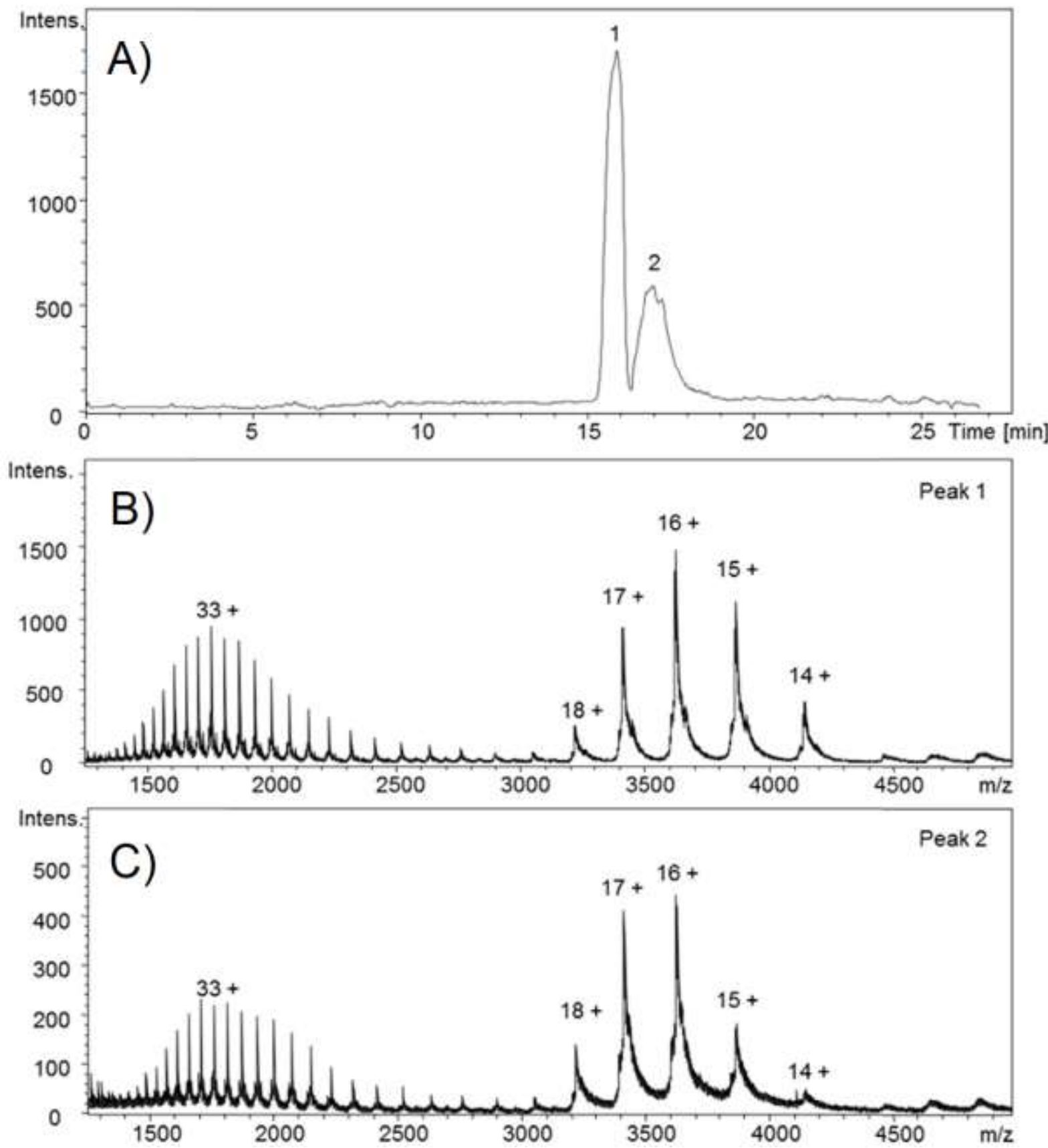